\title{
Capturability of 3D PPN against Lower-Speed Maneuvering Target for Homing Phase
}

\author{
Ke-Bo Li, Hyo-Sang Shin, Member IEEE, Antonios Tsourdos, and Min-Jea Tahk, Member IEEE
}

\begin{abstract}
The capturability of two-dimensional (2D) pure proportional navigation (PPN) guidance law against lower-speed arbitrarily maneuvering target for homing phase had been thoroughly analyzed by using the nonlinear output regulation (NOR) method before. However, due to the complexity of the three-dimensional (3D) relative kinematics, the NOR method has not been applied to the capturability analysis of 3D PPN, which leads to the capturability discrepancy of 2D PPN and its 3D extension. Thanks to the 3D relative kinematic equation between the missile and target established in the rotating line of sight (LOS) coordinate system, the capturability of 3D PPN against the lower-speed arbitrarily maneuvering target for the homing phase is restudied by extending the NOR method of 2D PPN to the 3D space. The necessary and sufficient condition for the missile guided by 3D PPN to intercept this type of target is obtained. It is proven that the capturability of 3D PPN is identical with that of 2D PPN.
\end{abstract}

Index Terms-3D PPN, capturability, nonlinear output regulation method, lower-speed maneuvering target

\section{INTRODUCTION}

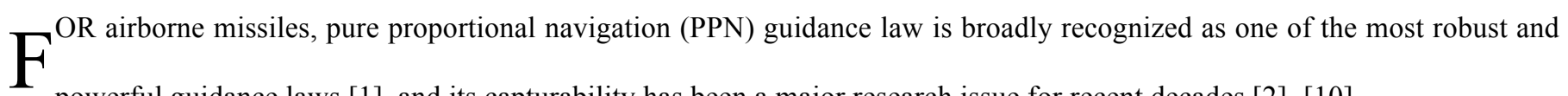
powerful guidance laws [1], and its capturability has been a major research issue for recent decades [2] [10].

Endoatmospheric targets are commonly categorized into lower-speed and higher-speed targets ${ }^{1}$. For the class of lower-speed targets, Guelman [2] [4] analyzed the capturability of PPN by using a qualitative method; Becker [5] obtained the closed-form solution of PPN based on the expansion theory of meromorphic function; Ghawghawe and Ghose [6] extended Guelman's method to the capturability analysis of PPN against the maneuvering target with a time-varying normal acceleration; Ha et al. [7] proposed a Lyapunov-like approach to analyze the performance of PPN against a randomly maneuvering target for the homing phase where

This work was co-supported by the National Natural Science Foundation of China (Grant Nos. 61690210(2017-2021) and 61690213(2017-2021)).K.-B. Li is

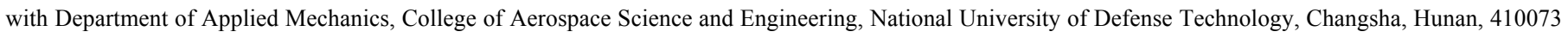
China, (e-mail: likeboreal@nudt.edu.cn).

H.-S. Shin, and A. Tsourdos are with the Institute of Aerospace Sciences, SATM, Cranfield University, College Road, Cranfield, Bedfordshire, MK43 0AL United Kingdom.

Min-Jea Tahk is with Department of Aerospace Engineering, KAIST, Daejeon, 305-701 Rep. of Korea.

${ }^{1}$ The target is called "lower-speed target", if its speed is smaller than the missile speed; else, it is called "higher-speed target". 
the missile is initially flying toward the target.

The above research is confined to two-dimensional (2D) space. Considering the cross coupling effect of pitch and yaw planes of missile, Song and Ha [8] extended the Lyapunov-like approach to the three-dimensional (3D) space, and analyzed the capturability, intercept time, line of sight rate convergence, and maximum commanded acceleration requirement of 3D PPN against lower-speed randomly maneuvering targets under the condition that the missile was initially flying toward the target. Based on the Lyapunov-like approach, Oh and Ha [9] proved that the missile guided by 3D PPN can always intercept a lower-speed arbitrarily maneuvering target with limited normal acceleration, when the navigation gain is large enough and the initial relative range is smaller than a certain value, no matter the missile is flying toward or away from the target initially.

The prior research only focuses on the case that the missile-to-target range is doomed to be strictly decreasing after a finite time and excludes the case when the relative range has a fluctuating time-profile caused by the target maneuver. By using the nonlinear output regulation (NOR) method, Oh [10] proved that, when the missile guided by 2D PPN was initially flying toward the target, the necessary and sufficient condition for the missile to intercept a lower-speed maneuvering target with an arbitrarily normal acceleration is a larger-than-one navigation gain, i.e., $N>1$.

Comparing [9] with [10] it can be seen that, the capturability discrepancy between 2D PPN and its 3D extension against the lower-speed arbitrarily maneuvering target is just for the case of $\rho \in[1 / \sqrt{2}, 1)$, where $\rho$ is the speed ratio of the target with respect to the missile. According to [9], for 3D PPN it requires $N$ to be larger than a constant which is a function of $\rho$ and the relative distance to be smaller than a constant which is a function of the target maximum acceleration, missile speed, $\rho$, and $N$. While for $2 \mathrm{D}$ PPN, the only requirement is $N>1$. According to numerical simulation results, for $3 \mathrm{D}$ PPN, $N>1$ is enough for intercepting lower-speed arbitrarily maneuvering targets. However, the theoretical proof is still missing. On the other hand, considering the complexity of the relative kinematic equation used in [9], the chance to prove it is slim.

Unlike previous literature, this paper investigates the capturability of 3D PPN against the lower-speed arbitrarily maneuvering target for the homing phase by using the relative kinematic equation established in the rotating line of sight (LOS) coordinate system [1], [11] [15], which simplifies the analysis process and removes the involvement of redundant variables. The rotating LOS coordinate system is similar to the modified polar coordinate system (MPC) in [16] [18] and is also called the LOS fixed coordinate system in Refs. [19] [22]. The NOR method in [10] is employed and extended to 3D space, where the nonlinear system is the 3D missile-target pursuit dynamics, the feedback controller is the 3D PPN guidance law, the output is the missile-to-target range, and the perturbation is the time-varying normal acceleration of the target. This paper will prove that, just like $2 \mathrm{D}$ PPN, $N>$ 1 is also the sufficient and necessary condition of 3D PPN to achieve zero miss distance.

The capturability of PPN against higher-speed targets has also been studied by some literature [18] [20], [22]. However, for the complexity of 3D relative motion between missile guided by PPN and higher-speed targets, this work hasn't been done completely 
and still needs further exploration.

\section{PRELIMINARIES}

The 3D pursuit situation (Fig. 1 in [8]) is shown in Fig. 1.

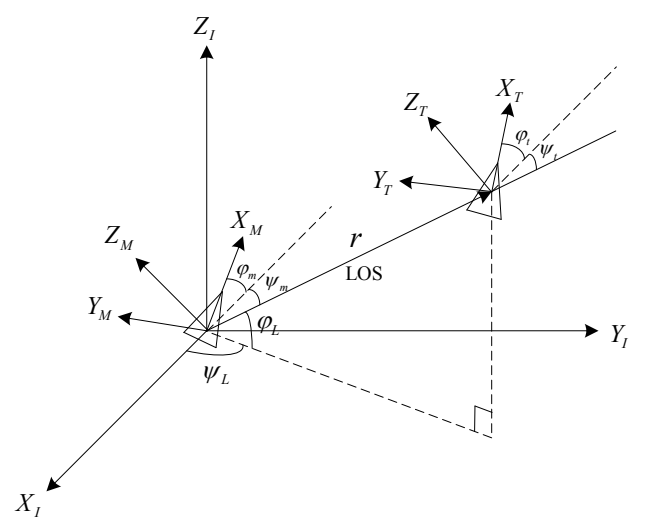

Fig. 1. The 3D pursuit geometry.

The assumptions adopted in [8] and [9] are also assumed to be valid in this note: 1) the missile and target are point masses; 2) the autopilot and seeker dynamics of the missile are fast enough to be neglected; 3) the angle-of-attack is small enough to be neglected; and 4) the speeds of the missile and target are constant. The relative kinematic equations ((1) (6) in [8] or (1) and (2) in[9]) are listed as below ${ }^{2}$

$$
\begin{gathered}
\dot{\boldsymbol{L}}=\boldsymbol{V}_{T}-\boldsymbol{V}_{M}=\ddot{r}_{L}+\boldsymbol{\Omega}_{L} \times \boldsymbol{L} \\
\boldsymbol{A}_{T}=a_{y t} \boldsymbol{j}_{T}+a_{z t} \boldsymbol{k}_{T}=\boldsymbol{\Omega}_{L} \times \boldsymbol{V}_{T}+\boldsymbol{\Omega}_{T} \times \boldsymbol{V}_{T} \\
\boldsymbol{A}_{M}=a_{y m} \boldsymbol{j}_{M}+a_{z m} \boldsymbol{k}_{M}=\boldsymbol{\Omega}_{L} \times \boldsymbol{V}_{M}+\boldsymbol{\Omega}_{M} \times \boldsymbol{V}_{M}
\end{gathered}
$$

where

$$
\begin{gathered}
\boldsymbol{L}=r \boldsymbol{i}_{L}, \quad \boldsymbol{V}_{T}=v_{t} \boldsymbol{i}_{T}, \quad \boldsymbol{V}_{M}=v_{m} \boldsymbol{i}_{M} \\
\boldsymbol{\Omega}_{L}=\dot{\psi}_{L} \sin \varphi_{L} \boldsymbol{i}_{L}-\dot{\varphi}_{L} \dot{j}_{L}+\dot{\psi}_{L} \cos \varphi_{L} \boldsymbol{k}_{L} \\
=\dot{\lambda}_{x} i_{L}+\dot{\lambda}_{y} \boldsymbol{j}_{L}+\dot{\lambda}_{z} \boldsymbol{k}_{L} \\
\boldsymbol{\Omega}_{T}=\dot{\psi}_{T} \sin \varphi_{T} \boldsymbol{i}_{T}-\dot{\varphi}_{T} \dot{j}_{T}+\dot{\psi}_{T} \cos \varphi_{T} \boldsymbol{k}_{T} \\
\boldsymbol{\Omega}_{M}=\dot{\psi}_{M} \sin \varphi_{M} \boldsymbol{i}_{M}-\dot{\varphi}_{M} \dot{j}_{M}+\dot{\psi}_{M} \cos \varphi_{M} \boldsymbol{k}_{M}
\end{gathered}
$$

It is further assumed that the missile autopilot dynamics are neglected and the yaw and pitch accelerations of the missile are equal to their commands, i.e.,

\footnotetext{
${ }^{2}$ Note that the nomenclature used in this note is the same as [9].
} 


$$
\begin{aligned}
\boldsymbol{A}_{M} & \approx \boldsymbol{A}_{M}^{C}=a_{y m} \boldsymbol{j}_{M}+a_{z m} \boldsymbol{k}_{M} \\
& =N v_{m}\left(-\dot{\lambda}_{y} \sin \varphi_{m} \sin \psi_{m}+\dot{\lambda}_{z} \cos \varphi_{m}\right) \boldsymbol{j}_{M} \\
& -N v_{m} \dot{\lambda}_{y} \cos \psi_{m} \boldsymbol{k}_{M}
\end{aligned}
$$

The 2D subspace $\left(Y_{L}, Z_{L}\right)$ of the LOS coordinate system is called LOS plane in this paper, as given in [9].

$\boldsymbol{i}_{L}, \boldsymbol{j}_{L}, \boldsymbol{k}_{L}$ can be expressed as

$$
\begin{aligned}
\boldsymbol{i}_{L} & =\cos \varphi_{L} \cos \psi_{L} \boldsymbol{i}_{I}+\cos \varphi_{L} \sin \psi_{L} \boldsymbol{j}_{I}+\sin \varphi_{L} \boldsymbol{k}_{I} \\
\boldsymbol{j}_{L} & =-\sin \psi_{L} \boldsymbol{i}_{I}+\cos \psi_{L} \boldsymbol{j}_{I} \\
\boldsymbol{k}_{L} & =-\sin \varphi_{L} \cos \psi_{L} \boldsymbol{i}_{I}-\sin \varphi_{L} \sin \psi_{L} \boldsymbol{j}_{I}+\cos \varphi_{L} \boldsymbol{k}_{I}
\end{aligned}
$$

while $\boldsymbol{i}_{M}, \boldsymbol{j}_{M}, \boldsymbol{k}_{M}$ can be expressed as

$$
\begin{aligned}
\boldsymbol{i}_{M} & =\cos \varphi_{m} \cos \psi_{m} \boldsymbol{i}_{L}+\cos \varphi_{m} \sin \psi_{m} \boldsymbol{j}_{L}+\sin \varphi_{m} \boldsymbol{k}_{L} \\
\boldsymbol{j}_{M} & =-\sin \psi_{m} \boldsymbol{i}_{L}+\cos \psi_{m} \boldsymbol{j}_{L} \\
\boldsymbol{k}_{M} & =-\sin \varphi_{m} \cos \psi_{m} \boldsymbol{i}_{L}-\sin \varphi_{m} \sin \psi_{m} \boldsymbol{j}_{L}+\cos \varphi_{m} \boldsymbol{k}_{L}
\end{aligned}
$$

Note that (10) still hold with index letters $M$ and $m$ substituted by $T$ and $t$, respectively.

Although the above equations can be used to analyze the capaturability of 3D PPN, however, as we can see, too many variables are involved. In the following content, the rotating LOS coordinate system will be introduced to reduce the complexity of the description of the 3D relative dynamics.

Since $\boldsymbol{\Omega}_{L}$ is the angular velocity of LOS, according to (5), taking the derivative of $\boldsymbol{i}_{L}$ with respect to time yields

$$
\begin{aligned}
\frac{\mathrm{d} \boldsymbol{i}_{L}}{\mathrm{~d} t} & =\boldsymbol{\Omega}_{L} \times \boldsymbol{i}_{L}=\left(\dot{\psi}_{L} \sin \varphi_{L} \boldsymbol{i}_{L}-\dot{\varphi}_{L} \boldsymbol{j}_{L}+\dot{\psi}_{L} \cos \varphi_{L} \boldsymbol{k}_{L}\right) \times \boldsymbol{i}_{L} \\
& =\dot{\psi}_{L} \cos \varphi_{L} \dot{j}_{L}+\dot{\varphi}_{L} \boldsymbol{k}_{L}
\end{aligned}
$$

From the above equation we can see that, the spin of LOS, i.e., " $\dot{\psi}_{L} \sin \varphi_{L} \boldsymbol{i}_{L}$ ”, make no change to LOS direction. Therefore, for 3D pursuit, the angular velocity vector of LOS excluding the $X_{L}$-component is more important, i.e.,

$$
\boldsymbol{\Omega}_{L O S}=-\dot{\varphi}_{L} \dot{j}_{L}+\dot{\psi}_{L} \cos \varphi_{L} \boldsymbol{k}_{L}=\dot{\lambda}_{y} \dot{j}_{L}+\dot{\lambda}_{z} \boldsymbol{k}_{L}
$$

If we denote

$$
\Omega_{L O S}=\sqrt{\left(\dot{\psi}_{L} \cos \varphi_{L}\right)^{2}+\dot{\varphi}_{L}^{2}}
$$

then,

$$
\boldsymbol{\Omega}_{L O S}=\Omega_{L O S} \boldsymbol{k}_{R}, \quad \boldsymbol{k}_{R}=\left(-\dot{\varphi}_{L} \dot{\boldsymbol{j}}_{L}+\dot{\psi}_{L} \cos \varphi_{L} \boldsymbol{k}_{L}\right) / \Omega_{L O S}
$$

If we further denote

$$
\boldsymbol{i}_{R}=\boldsymbol{i}_{L}, \quad \boldsymbol{j}_{R}=\boldsymbol{k}_{R} \times \boldsymbol{i}_{R}=\left(\dot{\psi}_{L} \cos \varphi_{L} \dot{j}_{L}+\dot{\varphi}_{L} \boldsymbol{k}_{L}\right) / \Omega_{L O S}
$$

then, $\boldsymbol{i}_{R}, \boldsymbol{j}_{R}, \boldsymbol{k}_{R}$ constitute the three axes of the rotating LOS coordinate system $\left(X_{R}, Y_{R}, Z_{R}\right)$.

The kinematic equation of $\left(X_{R}, Y_{R}, Z_{R}\right)$ can be deduced as 


$$
\frac{\mathrm{d}}{\mathrm{d} t}\left[\begin{array}{l}
\boldsymbol{i}_{R} \\
\boldsymbol{j}_{R} \\
\boldsymbol{k}_{R}
\end{array}\right]=\left[\begin{array}{ccc}
0 & \Omega_{L O S} & 0 \\
-\Omega_{L O S} & 0 & \Omega_{E P} \\
0 & -\Omega_{E P} & 0
\end{array}\right]\left[\begin{array}{l}
\boldsymbol{i}_{R} \\
\boldsymbol{j}_{R} \\
\boldsymbol{k}_{R}
\end{array}\right]
$$

where $\boldsymbol{\Omega}_{E P}=\Omega_{E P} \boldsymbol{i}_{R}$ is the angular velocity vector of the relative engagement plane (spanned by the relative position and velocity vectors) and $\Omega_{E P}$ the angular rate. The relative dynamic equation set in $\left(X_{R}, Y_{R}, Z_{R}\right)$ is shown as below

$$
\left\{\begin{aligned}
\ddot{r}-r \Omega_{L O S}^{2} & =\left(\boldsymbol{A}_{T} \cdot \boldsymbol{i}_{R}\right)-\left(\boldsymbol{A}_{M} \cdot \boldsymbol{i}_{R}\right) \\
r \dot{S}_{L O S}+2 \dot{r} \Omega_{L O S} & =\left(\boldsymbol{A}_{T} \cdot \boldsymbol{j}_{R}\right)-\left(\boldsymbol{A}_{M} \cdot \boldsymbol{j}_{R}\right) \\
r \Omega_{L O S} \Omega_{E P} & =\left(\boldsymbol{A}_{T} \cdot \boldsymbol{k}_{R}\right)-\left(\boldsymbol{A}_{M} \cdot \boldsymbol{k}_{R}\right)
\end{aligned}\right.
$$

The first two equations of (17) represent the relative motion between the missile and target in the engagement plane and are decoupled with the third one which describes the rotational principle of the engagement plane. For further information about (16) and (17), the reader is referred to Refs. [11] [14] .

Equation (8) can be rewritten as

$$
\begin{aligned}
\boldsymbol{A}_{M} & =N \boldsymbol{\Omega}_{L O S} \times \boldsymbol{V}_{M}=N v_{m} \Omega_{L O S}\left(\boldsymbol{k}_{R} \times \boldsymbol{i}_{M}\right) \\
& =N v_{m} \Omega_{L O S}\left[-\left(\boldsymbol{i}_{M} \cdot \boldsymbol{j}_{R}\right) \boldsymbol{i}_{R}+\left(\boldsymbol{i}_{M} \cdot \boldsymbol{i}_{R}\right) \boldsymbol{j}_{R}\right]
\end{aligned}
$$

The geometric relationship between $\boldsymbol{i}_{M}$ and $\boldsymbol{i}_{R}$ is shown in Fig. 2, and so is the geometric relationship between $\boldsymbol{i}_{T}$ and $\boldsymbol{i}_{R}$.

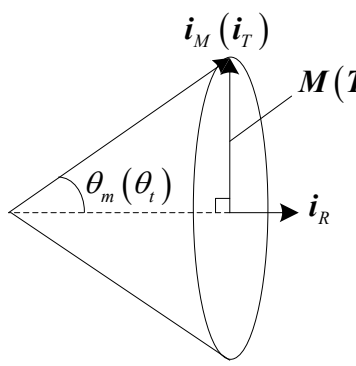

(a)

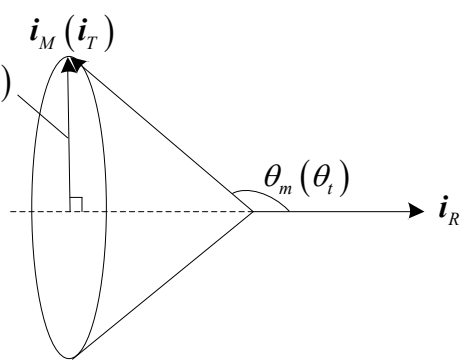

(b)

Fig. 2. Geometric relationship between the direction of missile (target) velocity and LOS.

From Fig. 2, the following relationships can be found,

$$
\boldsymbol{i}_{M} \cdot \boldsymbol{i}_{R}=\cos \theta_{m}, \quad \boldsymbol{i}_{T} \cdot \boldsymbol{i}_{R}=\cos \theta_{t}
$$

where $\theta_{m}$ is the angle between $\boldsymbol{i}_{M}$ and $\boldsymbol{i}_{R}$, and $\theta_{t}$ is the angle between $\boldsymbol{i}_{T}$ and $\boldsymbol{i}_{R}$. In some literature, $\theta_{m}$ is called lead angle.

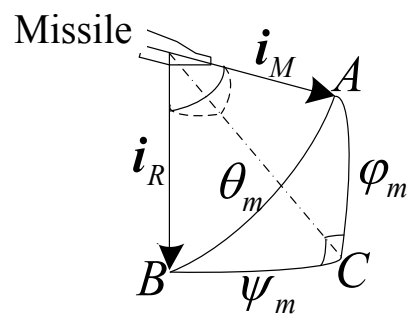


Fig. 3. Geometric relationship between $\varphi_{m}, \psi_{m}$, and $\theta_{m}$.

The geometric relationship between $\varphi_{m}, \psi_{m}$, and $\theta_{m}$ is shown in Fig. 3, where $\triangle \mathrm{ABC}$ is a spherical right-angled triangle and $\angle \mathrm{C}$ $=\pi / 2$. Note that geometric relationships in Fig. 3 still hold with index letter $m$ substituted by $t$. Then we have

$$
\cos \theta_{m}=\cos \varphi_{m} \cos \psi_{m}
$$

The following situation is mainly considered, i.e.,

$$
\left|\varphi_{m}(0)\right|<\pi / 2, \quad\left|\psi_{m}(0)\right|<\pi / 2
$$

which represents that the missile is initially flying toward the target. [9] also discussed the situation when (21) was not satisfied. Since the new theoretical findings of capaturability of 3D PPN are under (21), we mainly discuss this situation in this paper. Considering the definition domain of $\theta_{m},(20)$ and (21) lead to

$$
\theta_{m}(0) \in(-\pi / 2, \pi / 2)
$$

Although (22) is valid by definition, in this paper the case of $\theta_{m}(0) \in[0, \pi / 2)$ is only considered. The analysis process of the case of $\theta_{m}(0) \in(-\pi / 2,0]$ is quite similar to the case of $\theta_{m}(0) \in[0, \pi / 2)$, and hence it is omitted for the length of the paper.

Define the vectors $\boldsymbol{M}$ and $\boldsymbol{T}$ by

$$
\boldsymbol{M}=\boldsymbol{i}_{M}-\left(\boldsymbol{i}_{M} \cdot \boldsymbol{i}_{R}\right) \boldsymbol{i}_{R}, \quad \boldsymbol{T}=\boldsymbol{i}_{T}-\left(\boldsymbol{i}_{T} \cdot \boldsymbol{i}_{R}\right) \boldsymbol{i}_{R}
$$

as shown in Fig. 2. Then

$$
|\boldsymbol{M}|=\sin \theta_{m}, \quad|\boldsymbol{T}|=\sin \theta_{t}
$$

The angle between $\boldsymbol{M}$ and $\boldsymbol{T}$ is denoted as $\Theta$ :

$$
\cos \Theta=\frac{\boldsymbol{M} \cdot \boldsymbol{T}}{|\boldsymbol{M}||\boldsymbol{T}|}=\frac{\boldsymbol{i}_{M} \cdot \boldsymbol{i}_{T}-\cos \theta_{m} \cos \theta_{t}}{\sin \theta_{m} \sin \theta_{t}}
$$

Note that, the definition of $\Theta$ is only valid when $|\boldsymbol{M}|$ and $|\boldsymbol{T}| \neq 0$. If not, $\Theta$ will become the angle between a nonzero vector and a zero vector or the angle between two zero vectors, and in this case, we define

$$
\Theta(t)=0, \text { if } \sin \theta_{m}(t)=0 \text { or } \sin \theta_{t}(t)=0
$$

Throughout this paper, the target speed is assumed to satisfy

$$
1 / \sqrt{2} \leq \rho=v_{t} / v_{m}<1
$$

From the above definitions and assumptions, the following kinematic equations can be obtained:

$$
\begin{gathered}
v_{r}=\dot{r}=\left(\boldsymbol{V}_{T}-\boldsymbol{V}_{M}\right) \cdot \boldsymbol{i}_{R}=v_{m}\left(\rho \cos \theta_{t}-\cos \theta_{m}\right) \\
v_{\theta}=r \Omega_{L O S}=\left(\boldsymbol{V}_{T}-\boldsymbol{V}_{M}\right) \cdot \boldsymbol{j}_{R}=v_{m}|\rho \boldsymbol{T}-\boldsymbol{M}|
\end{gathered}
$$


where $v_{r}$ is the closing speed and $v_{\theta}$ the transversal relative speed.

Finally, the target acceleration $\boldsymbol{A}_{T}$ is assumed to satisfy the following condition:

$$
\theta_{t} \in C^{P}
$$

where $C^{P}$ denotes the set of all piecewise continuous functions defined on $t \geq 0$. This assumption was firstly adopted in [10] in 2D space, where $\theta_{t}$ was a 2D angle with different definition domain, and is much more general than the assumption of the boundedness of $\left|\boldsymbol{A}_{T}\right|$.

\section{MAIN ReSUlts}

According to the authors' knowledge, when the missile was initially flying toward a lower-speed target satisfying (27), the least conservative results of capturability of 3D PPN were given in (Theorem 2 in [9]). While under same conditions, the least conservative results of capturability of 2D PPN were given in (Theorem 1 in [10]).

For 2D PPN, the capture condition under (27) is [10]

$$
N>1
$$

while for 3D PPN, the counterpart is

$$
N>1+\sqrt{2 \rho^{2}-1} / \rho, \quad r(0)<\frac{\rho^{2} v_{m}^{2}}{\alpha^{\prime}}\left[\rho / \sqrt{2 \rho^{2}-1}-\operatorname{sgn}(N-2)\right]\left[N-1+\operatorname{sgn}(N-2) \sqrt{2 \rho^{2}-1} / \rho\right]\left(1-\rho^{2}\right)^{\frac{1}{2(N-1)}}
$$

where $\alpha^{\prime}$ is the upper bound of $\left|\boldsymbol{A}_{T}\right|$ and is limited. It can be seen that the capture condition of 3D PPN given in (32) is more conservative than that of $2 \mathrm{D}$ PPN, i.e., $N>1$.

However, according to numerical simulation results, when $1<N \leq 1+\sqrt{2 \rho^{2}-1} / \rho$ and $r(0)$ is random, the missile guided by $3 \mathrm{D}$ PPN can still capture lower-speed maneuvering target with arbitrary normal acceleration, which contradicts with (Theorem 2 in [10]). This motivates us to restudy the capturability of 3D PPN.

Our purpose is to prove that the necessary and sufficient condition of 3D PPN guaranteeing the following property:

$$
\min _{\tau<t} r(\tau)=0, \quad \text { as } \quad t \rightarrow \infty
$$

i.e., $r \rightarrow 0$ at a finite time, under (22), (27), and (30), is still $N>1$.

Theorem 1: Under the assumption of (30) and the conditions of (22) and (27), the system represented in (1) (10) has the property of (33), i.e., $r \rightarrow 0$ at a finite time, if and only if $N>1$ is satisfied.

The necessity part of Theorem 1 can be easily proven. Actually, it has already been discussed in [9] that there exist some pursuit situations that the missile guided by $3 \mathrm{D}$ PPN with $N \leq 1$ can miss a lower-speed maneuvering target with an appropriate acceleration profile, which completes the proof of the necessity part. Therefore, we mainly prove the sufficiency part of this theorem. Before proving, some lemmas need to be introduced firstly, some of which are from [9] and [10]. 
It can be easily found that, using (20), (Lemma 1 in [9]) can be simplified into the following lemma, and hence the proof is omitted.

Lemma 1: If $N>1$ is satisfied, then the 3D PPN guidance law guarantees that

$$
\sin \theta_{m}(t) \leq \max \left\{\sin \theta_{m}(0), \rho\right\}, \quad \forall t \geq 0
$$

A discursion could be deduced from Lemma 1 .

Discursion 1: If $N>1$ is satisfied, then the 3D PPN guidance law guarantees that

$$
\sin \theta_{m}(t) \leq \rho, \quad \forall t \geq t_{c}
$$

where $t_{c}$ is defined as

$$
t_{c}=:\left\{\begin{array}{lll}
0, & \text { if } & \theta_{m}(0) \leq \sin ^{-1} \rho \\
\left\{t>0 \mid \sin \theta_{m}(t)=\rho\right\}, & \text { if } & \theta_{m}(0)>\sin ^{-1} \rho
\end{array}\right.
$$

Proof: Taking the derivative of $\cos \theta_{m}$ with respect to time yields

$$
\begin{aligned}
\frac{\mathrm{d}\left(\cos \theta_{m}\right)}{\mathrm{d} t} & =\frac{\mathrm{d}\left(\boldsymbol{i}_{M} \cdot \boldsymbol{i}_{R}\right)}{\mathrm{d} t}=\frac{\boldsymbol{A}_{M}}{v_{m}} \cdot \boldsymbol{i}_{R}+\boldsymbol{i}_{M} \cdot\left(\Omega_{L O S} \boldsymbol{j}_{R}\right) \quad \forall t>0 \\
& =(1-N) \Omega_{L O S}\left(\boldsymbol{i}_{M} \cdot \boldsymbol{j}_{R}\right)
\end{aligned}
$$

Since

$$
\boldsymbol{j}_{R}=\frac{\left(\boldsymbol{V}_{T}-\boldsymbol{V}_{M}\right)-\left[\left(\boldsymbol{V}_{T}-\boldsymbol{V}_{M}\right) \cdot \boldsymbol{i}_{R}\right] \boldsymbol{i}_{R}}{v_{\theta}}=\frac{v_{m}(\rho \boldsymbol{T}-\boldsymbol{M})}{r \Omega_{L O S}}
$$

Equation (37) can be rewritten into

$$
\begin{aligned}
\dot{\theta}_{m} & =-v_{m}(N-1)\left(\sin \theta_{m}-\rho \sin \theta_{t} \cos \Theta\right) / r \quad \forall t>0 \\
& \leq-v_{m}(N-1)\left(\sin \theta_{m}-\rho \sin \theta_{t}\right) / r,
\end{aligned}
$$

Hence, according to (39) it can be deduced that $\dot{\theta}_{m}<0$ under $\theta_{m}(t)>\sin ^{-1} \rho$. Then, (35) can be easily proven.

Lemma 2: If $N>1$ is satisfied and $\rho=1 / \sqrt{2}$, then 3D PPN guidance law guarantees $\min _{\tau<t} r(\tau)=0$, as $t \rightarrow \infty$.

Proof: According Lemma 1, when $\rho=1 / \sqrt{2}$, we have $\cos \theta_{m}(t) \geq \sqrt{1-\rho^{2}}=1 / \sqrt{2}$ for $t \geq t_{c}$. Then, according to (28) yields

$$
\dot{r} \leq v_{m}\left(\cos \theta_{t}-1\right) / \sqrt{2}, \quad t \geq t_{c}
$$

which means $\dot{r}=0$ only happens when

$$
\theta_{t}(t) \equiv 0, \quad t \geq t_{c}
$$

Substituting (41) into (39) leads to

$$
\dot{\theta}_{m}=-v_{m}(N-1) \sin \theta_{m} / r, \quad\left\{t \geq t_{c} \mid \theta_{t}(t) \equiv 0\right\}
$$


which together with $N>1$ indicates that $\theta_{m}(t)$ reaches zero ultimately under (41). Then, this together with (28) and (41) leads to $\dot{r}(t)<0$ at steady state under (41). Therefore, it is guaranteed that $r \rightarrow 0$ at a finite time.

Lemma 3: If $N>1$ is satisfied, for

$$
\theta_{m}(t) \in\left[0, \cos ^{-1} \rho\right), \quad \forall t \geq 0
$$

then

$$
\dot{r}(t)<0, \quad \forall t \geq 0
$$

Proof: According to (28), if (43) holds, we have

$$
\dot{r}=v_{m}\left(\rho \cos \theta_{t}-\cos \theta_{m}\right)<v_{m} \rho\left(\cos \theta_{t}-1\right) \leq 0, \quad \forall t \geq 0
$$

which leads to (44).

According to Lemma 1 3, when $t \geq t_{c}, \dot{r}>0$ only happens in the region of $S$, which is defined by

$$
S=\left\{t \in\left[t_{c},+\infty\right) \mid \theta_{m}(t) \in\left[\cos ^{-1} \rho, \sin ^{-1} \rho\right]\right\}
$$

where $1 / \sqrt{2}<\rho<1$.

In [10], the nonlinear output regulation (NOR) method was directly applied to 2D PPN with $\theta_{m}(t) \in\left[0\right.$, $\left.\sin ^{-1} \rho\right]$. However, in this paper, we find the more specific domain of $\theta_{m}$ where the NOR method should be applied, i.e., (46). This will help the reader to understand the relative kinematics between the missile guided by PPN and the lower-speed arbitrarily maneuvering target better. Besides, we can see that, according to (46), as $\rho$ increases in $(1 / \sqrt{2}, 1), S$ is enlarged from $\theta_{m}(t) \in(\pi / 4, \pi / 4)$ to $\theta_{m}(t) \in(0, \pi / 2)$. For the research of the property of $r(t)$ in $S$, we need to introduce the following four Lemmas. The following Lemma 4 is actually (Lemma 2 in [9]) with simplified and more detailed expression.

Lemma 4: In addition to $N>1$, suppose the following inequality holds for a closed time interval $\left[t_{0}, t_{1}\right]$,

$$
\sin \theta_{m}(t) \geq \rho \sin \theta_{t}(t), \quad t \in\left[t_{0}, t_{1}\right]
$$

Then, 3D PPN guarantees that

$$
\frac{r\left(t_{1}\right)}{r\left(t_{0}\right)} \leq\left\{\begin{array}{l}
{\left[\frac{\rho \cos \theta_{m}\left(t_{1}\right)+\sqrt{1-\rho^{2}} \sin \theta_{m}\left(t_{1}\right)}{\rho \cos \theta_{m}\left(t_{0}\right)+\sqrt{1-\rho^{2}} \sin \theta_{m}\left(t_{0}\right)}\right]^{\frac{1}{N-1}}, \text { if } \cos \theta_{m}\left(t_{1}\right) \leq \rho} \\
{\left[\rho \cos \theta_{m}\left(t_{0}\right)+\sqrt{1-\rho^{2}} \sin \theta_{m}\left(t_{0}\right)\right]^{-\frac{1}{N-1}} \text { if } \cos \theta_{m}\left(t_{1}\right)>\rho} \\
\leq\left[1-\rho^{2}\right]^{-\frac{1}{2(N-1)}},
\end{array}\right.
$$

where. $\theta_{m}\left(t_{0}\right) \geq \theta_{m}\left(t_{1}\right)$.

According to Lemma 4, it can also be deduced that, for 3D PPN, ((44) in [9]) 


$$
r(t) \leq r_{\max } \leq r_{0}\left[1-\rho^{2}\right]^{-\frac{1}{2(N-1)}}, \quad t \geq t_{0}
$$

The following Lemma 5 is about the opposite situation of Lemma 4.

Lemma 5: In addition to $N>1$, suppose the following inequality holds for a closed time interval $\left[t_{0}, t_{1}\right]$,

$$
\sin \theta_{m}(t) \leq \rho \sin \theta_{t}(t), \quad t \in\left[t_{0}, t_{1}\right]
$$

Then, 3D PPN guarantees that

$$
\frac{r\left(t_{1}\right)}{r\left(t_{0}\right)} \leq\left[\frac{\rho \cos \theta_{m}\left(t_{1}\right)-\sqrt{1-\rho^{2}} \sin \theta_{m}\left(t_{1}\right)}{\rho \cos \theta_{m}\left(t_{0}\right)-\sqrt{1-\rho^{2}} \sin \theta_{m}\left(t_{0}\right)}\right]^{\frac{1}{N-1}}
$$

where $\theta_{m}\left(t_{0}\right) \leq \theta_{m}\left(t_{1}\right)$.

Proof: According to (50), we have

$$
\begin{aligned}
\sin ^{2} \theta_{m} & \leq \rho^{2} \sin ^{2} \theta_{t} \Rightarrow 1-\cos ^{2} \theta_{m} \leq \rho^{2}-\rho^{2} \cos ^{2} \theta_{t} \\
& \Rightarrow \rho^{2} \cos ^{2} \theta_{t}-\cos ^{2} \theta_{m} \leq \rho^{2}-1<0
\end{aligned}
$$

and

$$
\dot{r}(t) \leq v_{m}\left[\rho \cos \theta_{t}(t)-\sqrt{1-\rho^{2} \sin ^{2} \theta_{t}(t)}\right]=v_{m} g\left[\theta_{t}(t)\right]
$$

where $g\left[\theta_{t}(t)\right]$ is a continuous function of time and also a monotonic decreasing function of $\theta_{t}(t)$. Therefore,

$$
\dot{r}(t) \leq v_{m} g\left[\theta_{t}(t)\right]_{\theta_{t}(t)=0}=v_{m}(\rho-1)<0
$$

Then, according to (39) and (50),

$$
0 \leq \frac{\mathrm{d} \theta_{m}}{\mathrm{~d} t} \leq-\frac{v_{m}}{r}(N-1)\left(\sin \theta_{m}-\rho \sin \theta_{t}\right), \quad t \in\left[t_{0}, t_{1}\right]
$$

which can be rewritten into

$$
-\frac{1}{v_{m}(N-1)\left(\sin \theta_{m}-\rho \sin \theta_{t}\right)} \frac{\mathrm{d} \theta_{m}}{\mathrm{~d} t} \leq \frac{1}{r}, \quad t \in\left[t_{0}, t_{1}\right]
$$

Considering (28), (54), and (56), we have

$$
\frac{\dot{r}}{r} \leq-\frac{\rho \cos \theta_{t}-\cos \theta_{m}}{(N-1)\left(\sin \theta_{m}-\rho \sin \theta_{t}\right)} \frac{\mathrm{d} \theta_{m}}{\mathrm{~d} t}, \quad t \in\left[t_{0}, t_{1}\right]
$$

By taking the derivative of RHS of (57) with respective to $\theta_{t}$ and considering (50), we can easily see that $\dot{r} / r$ is maximized by

$$
\left\{\begin{array}{l}
\cos \theta_{t}=\rho \cos \theta_{m}-\sqrt{1-\rho^{2}} \sin \theta_{m} \\
\sin \theta_{t}=\rho \sin \theta_{m}+\sqrt{1-\rho^{2}} \cos \theta_{m}
\end{array}\right.
$$

Then, 


$$
\frac{\dot{r}}{r} \leq-\frac{\rho \sin \theta_{m}+\sqrt{1-\rho^{2}} \cos \theta_{m}}{(N-1)\left(\rho \cos \theta_{m}-\sqrt{1-\rho^{2}} \sin \theta_{m}\right)} \frac{\mathrm{d} \theta_{m}}{\mathrm{~d} t}, \quad t \in\left[t_{0}, t_{1}\right]
$$

Taking the integral of (59), the following inequality holds

$$
\int_{t_{0}}^{t_{1}} \frac{\dot{r}}{r} \leq \int_{t_{0}}^{t_{1}}-\frac{\rho \sin \theta_{m}+\sqrt{1-\rho^{2}} \cos \theta_{m}}{(N-1)\left(\rho \cos \theta_{m}-\sqrt{1-\rho^{2}} \sin \theta_{m}\right)} \frac{\mathrm{d} \theta_{m}}{\mathrm{~d} t}
$$

The above inequality can be rewritten as

$$
\ln \frac{r\left(t_{1}\right)}{r\left(t_{0}\right)} \leq \frac{1}{N-1} \ln \frac{\rho \cos \theta_{m}\left(t_{1}\right)-\sqrt{1-\rho^{2}} \sin \theta_{m}\left(t_{1}\right)}{\rho \cos \theta_{m}\left(t_{0}\right)-\sqrt{1-\rho^{2}} \sin \theta_{m}\left(t_{0}\right)}
$$

Let

$$
h(t)=\rho \cos \theta_{m}(t)-\sqrt{1-\rho^{2}} \sin \theta_{m}(t)
$$

Then,

$$
\mathrm{d}[h(t)] / \mathrm{d} \theta_{m}(t)=-\rho \sin \theta_{m}(t)-\sqrt{1-\rho^{2}} \cos \theta_{m}(t)<0
$$

Therefore, (51) is the direct consequence of (61). Besides, according to (55),

$$
\theta_{m}\left(t_{0}\right) \leq \theta_{m}\left(t_{1}\right)
$$

Then, Lemma 5 is proved

For $S$ defined in (46), if $S \neq \varnothing$ holds, since $\dot{r}$ is a continuous function of time, there must exist at most a countable infinite number of subintervals $I_{i, i=1,2, \ldots}$ of $S$ such that

$$
\left\{\begin{array}{cl}
I_{i}=\left[b_{i}, e_{i}\right], & b_{i}<e_{i} \leq b_{i+1} \\
I_{i} \cap I_{j}=\varnothing, \quad \text { whenever } i \neq j \\
\bigcup_{i=1}^{n} I_{i}=S
\end{array}\right.
$$

where $n=1,2,3 \ldots$ is the number of $I_{i}$. According to (46), (65), and Lemma 3, we have,

$$
t_{c}=b_{1}, \quad r\left(e_{i}\right) \geq r\left(b_{i+1}\right)
$$

which can be shown in Fig. 4. Without loss of generality, we assume $\theta_{m}(0)>\sin ^{-1} \rho$ in Fig. 4 .

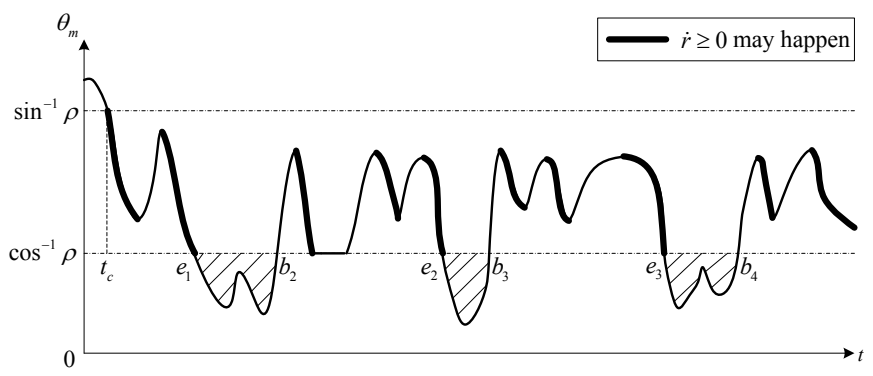


Fig. 4. The sketch of $\theta_{m}(t)$.

In Fig. 4, the shadowed area is the region of $\theta_{m}(t) \in\left[0, \cos ^{-1} \rho\right)$ where $\dot{r}(t)<0$, and the thick monotonic lines represent the region of $\left\{\theta_{m}(t) \in S \mid \dot{\theta}_{m}(t)<0\right\}$ where $\dot{r}(t) \geq 0$ may happen.

For each $I_{i}$, we can find time sequence $U_{i}=\left\{\tau_{i 0}, \tau_{i 1}, \tau_{i 2}, \ldots\right\}$

which is at most countable infinite such that for all $j=0,1,2, \ldots$,

$$
\left\{\begin{array}{c}
\tau_{i j}<\tau_{i(j+1)} \\
{\left[\theta_{m}\left(\tau_{i(j+1)}\right)-\theta_{m}\left(\tau_{i j}\right)\right]\left[\theta_{m}\left(\tau_{i(j+2)}\right)-\theta_{m}\left(\tau_{i(j+1)}\right)\right]<0} \\
{\left[\theta_{m}\left(\tau_{i(j+1)}\right)-\theta_{m}\left(\tau_{i j}\right)\right] \dot{\theta}_{m}(t) \geq 0, \quad t \in\left[\tau_{i j}, \tau_{i(j+1)}\right]}
\end{array}\right.
$$

where $k_{i}$ is the number of $\tau_{i j}$ in $U_{i}$.

Now, the following function can be introduced,

$$
\Phi\left(t_{1}, t_{2}\right)=\left\{\begin{array}{l}
{\left[\frac{\rho \cos \theta_{m}\left(t_{2}\right)-\sqrt{1-\rho^{2}} \sin \theta_{m}\left(t_{2}\right)}{\rho \cos \theta_{m}\left(t_{1}\right)-\sqrt{1-\rho^{2}} \sin \theta_{m}\left(t_{1}\right)}\right]^{\frac{1}{N-1}}, \quad \text { if } \theta_{m}\left(t_{1}\right)<\theta_{m}\left(t_{2}\right) \leq \sin ^{-1} \rho} \\
1, \quad \text { if } \theta_{m}\left(t_{1}\right)=\theta_{m}\left(t_{2}\right) \\
{\left[\frac{\rho \cos \theta_{m}\left(t_{2}\right)+\sqrt{1-\rho^{2}} \sin \theta_{m}\left(t_{2}\right)}{\rho \cos \theta_{m}\left(t_{1}\right)+\sqrt{1-\rho^{2}} \sin \theta_{m}\left(t_{1}\right)}\right]^{\frac{1}{N-1}}, \text { if } \theta_{m}\left(t_{2}\right)<\theta_{m}\left(t_{1}\right) \leq \sin ^{-1} \rho}
\end{array}\right.
$$

which is an extension of $((16)$ in [10]) in 3D space. And $\Phi$ has the following property ((46) in [10]):

$$
\Phi \leq\left[1-\rho^{2}\right]^{-\frac{1}{2(N-1)}}
$$

The following Lemma 6 shows an important property of $U_{i}$ and $\Phi$.

Lemma 6: There exists a sequence $\left\{\phi_{i k}, k=0,1,2,3, \ldots\right\}$ that

$$
\begin{gathered}
\phi_{i 0}=1, \quad 0<\phi_{i k}<1, \quad k=1,2,3 \ldots \\
\prod_{k=0}^{j} \Phi\left(\tau_{i k}, \tau_{i(k+1)}\right)=\Phi\left(\tau_{i 0}, \tau_{i(j+1)}\right) \prod_{k=0}^{j} \phi_{i k}
\end{gathered}
$$

where $\Phi\left(\tau_{i k}, \tau_{i(k+1)}\right)$ is defined by $(68)$ and $\left\{\phi_{i k}, k=0,1,2,3, \ldots\right\}$ is defined as follows,

$$
\phi_{i k}=\left\{\begin{array}{l}
\Phi\left(\tau_{i(k+1)}, \tau_{i k}\right) \Phi\left(\tau_{i k}, \tau_{i(k+1)}\right), \quad \text { if } \quad \theta_{m}\left(\tau_{i(k+1)}\right) \in J\left(\tau_{i 0}, \tau_{i k}\right) \\
\Phi\left(\tau_{i 0}, \tau_{i k}\right) \Phi\left(\tau_{i k}, \tau_{i 0}\right), \quad \text { otherwise }
\end{array}\right.
$$

and 


$$
J\left(t_{1}, t_{2}\right)=\left[\min \left\{\theta_{m}\left(t_{1}\right), \theta_{m}\left(t_{2}\right)\right\}, \max \left\{\theta_{m}\left(t_{1}\right), \theta_{m}\left(t_{2}\right)\right\}\right]
$$

Lemma 6 is an extension of (Lemma 2 in [10]) in 3D space for each $U_{i}$ of $S$. And hence the proof is omitted.

The upper bound of the missile-to-target range $r(t)$ in $I_{i}$ is identified in Lemma 7.

Lemma 7: Suppose that $N>1$ holds. Then, the 3D PPN guidance law guarantees that

$$
r\left(\tau_{n(j+1)}\right) / r\left(\tau_{10}\right) \leq \Phi\left(\tau_{10}, \tau_{n(j+1)}\right) \prod_{i=1}^{n} \prod_{k=0}^{\substack{k_{i}-1, i \neq n \\ j, i=n}} \phi_{i k}
$$

where $\left\{\phi_{i k}, k=0,1,2,3, \ldots\right\}$ satisfies $(70)$.

Lemma 7 is similar to (Lemma 3 in [10]) and hence the proof is omitted.

Now, we are ready to prove the sufficient part of Theorem 1. In the following proof, most equations from (Proof of the sufficient part of Theorem 1 in [10]) will be employed, since the method used in this note is an extension of the NOR method proposed in [10]. However, variables concerned here are in 3D space, and a new angle between the missile velocity and target velocity in the LOS plane, i.e., $\Theta$, is used, which helps to make a clear comprehension of the $3 \mathrm{D}$ relative motion principles between the missile and target.

Proof (of the sufficient part of Theorem 1): We firstly deal with the condition that $S$ is bounded. There are two situations under this condition. The first one is

$$
\theta_{m}(t) \in\left[0, \cos ^{-1} \rho\right), \quad t>T_{1}
$$

where $T_{1}$ is the last time that $\theta_{m}(t) \in S$. Then, according to Lemma 3,

$$
\dot{r}(t)<0, \quad \forall t \geq T_{1}
$$

which means $r \rightarrow 0$ at a finite time can be guaranteed.

The second one is

$$
\theta_{m}(t) \in\left[\cos ^{-1} \rho, \sin ^{-1} \rho\right], \quad t>T_{2}
$$

where $T_{2}$ is the last time that $\theta_{m}(t) \in\left[0, \cos ^{-1} \rho\right]$. Then, $T_{2} \rightarrow \tau_{n 0}$.

Let $\tau_{n j^{*}}$ be the last element of $S$. Since $U_{i}=\left\{\tau_{i 0}, \tau_{i 1}, \tau_{i 2}, \ldots\right\}$ is at most countable infinite, we can let $\tau_{n j^{*}}=+\infty$ without any loss of generality. Then, we can see from $(67)$ that $\theta_{m}(t)$ keeps either increasing or decreasing on $\left[\tau_{n\left(j^{*}-1\right)},+\infty\right)$. According to $(77), \theta_{m}(t)$ is bounded. Hence, we can say that $\dot{\theta}_{m}(t) \rightarrow 0$ as $t \rightarrow \infty$. This, along with (39) and (49), indicates that, for any $\varepsilon \in(0,1-\rho)$,

$$
\left|\sin \theta_{m}-\rho \sin \theta_{t} \cos \Theta\right| \leq \varepsilon, \quad t \in\left[T_{3},+\infty\right)
$$

where $T_{3}>\tau_{n\left(j^{*}-1\right)}$ is a constant. It can be deduced from (78) that, 


$$
\begin{aligned}
\sin ^{2} \theta_{m}-\rho^{2} \sin ^{2} \theta_{t} & \leq \varepsilon\left(\sin \theta_{m}+\rho \sin \theta_{t}\right) \\
& \leq \varepsilon(1+\rho)
\end{aligned} \quad t \in\left[T_{3},+\infty\right)
$$

i.e.,

$$
\rho^{2} \cos ^{2} \theta_{t}-\cos ^{2} \theta_{m} \leq \rho^{2}-1+\varepsilon(1+\rho)<0, \quad t \in\left[T_{3},+\infty\right)
$$

the above equation along with (28) leads to

$$
\begin{aligned}
\dot{r} & \leq v_{m}\left(\rho\left|\cos \theta_{t}\right|-\cos \theta_{m}\right) \leq v_{m} \frac{\left(\rho^{2} \cos ^{2} \theta_{t}-\cos ^{2} \theta_{m}\right)}{\rho\left|\cos \theta_{t}\right|+\cos \theta_{m}} \\
& \leq \frac{v_{m}\left[\rho^{2}-1+\varepsilon(1+\rho)\right]}{\rho+1} \leq v_{m}(\rho-1+\varepsilon)<0, \quad t \in\left[T_{3},+\infty\right)
\end{aligned}
$$

which indicates that $r \rightarrow 0$ at a finite time is guaranteed.

Then, we discuss the situation that $S$ is unbounded. We prove Theorem 1 under this condition by contradiction. The following proof will start from an assumption that $r$ is lower-bounded, i.e.,

$$
r(t) \geq r_{l b} \quad \forall t \geq t_{c}=0
$$

where subscript "lb" means "lower bound".

This assumption will be explained to be a sufficient condition for $\theta_{m}(t)$ to converge to a constant. In turn, it will be proven that, under this condition, $r(t)$ will converge to zero as $t \rightarrow \infty$. This contradicts (82). Then, $r \rightarrow 0$ at a finite time can be valid under this situation.

According to (39) and (82), $\dot{\theta}_{m}(t)$ is bounded by the following inequality,

$$
\left|\dot{\theta}_{m}\right| \leq v_{m}(N-1)(1+\rho) / r_{l b}
$$

Then, for a given constant $\alpha \in(\rho, 1)$ and

$$
\tau \in[t, t+\delta],\left\{\begin{array}{l}
\forall t \geq T_{2} \\
\forall \delta \in\left(0, \delta^{*}\right]
\end{array}\right.
$$

where $\delta^{*}$ is a positive constant satisfies

$$
\delta^{*} \leq \frac{r_{l b}}{v_{m}(N-1)(1+\rho)} \min \left\{\sin ^{-1}\left(\frac{\sqrt{1-\rho^{2}}}{\alpha}\right)-\cos ^{-1} \rho, \sin ^{-1} \rho-\sin ^{-1}(\alpha \rho), \quad \tan ^{-1}\left[\frac{(1-\alpha)}{(1+\alpha)} \frac{\rho}{\sqrt{1-\rho^{2}}}\right]\right\}
$$

it has

$$
\left\{\begin{array}{l}
\min \left\{\sin \theta_{m}\right\}=\sin \left(\min \left\{\theta_{m}\right\}\right) \\
\max \left\{\sin \theta_{m}\right\}=\sin \left(\max \left\{\theta_{m}\right\}\right)
\end{array}\right.
$$

for $\theta_{m}(t) \in\left[\cos ^{-1} \rho, \sin ^{-1} \rho\right]$ where $t \geq T_{2}$. (86) leads to 


$$
\frac{\min _{\tau \in[t, t+\delta]}\left\{\sin \theta_{m}\right\}}{\max _{\tau \in[t, t+\delta]}\left\{\sin \theta_{m}\right\}}=\frac{\sin \left(\min _{\tau \in[t, t+\delta]}\left\{\theta_{m}\right\}\right)}{\sin \left(\max _{\tau \in[t, t+\delta]}\left\{\theta_{m}\right\}\right)}
$$

For $t \geq T_{2}$, it further has

$$
\begin{aligned}
& \min _{\tau \in[t, t+\delta]}\left\{\theta_{m}\right\}=\cos ^{-1} \rho, \quad \text { or } \quad \theta_{m}(t)-\frac{v_{m}(N-1)(1+\rho)}{r_{l b}} \delta \geq \theta_{m}(t)-\frac{v_{m}(N-1)(1+\rho)}{r_{l b}} \delta^{*} \\
& \max _{\tau \in[t, t+\delta]}\left\{\theta_{m}\right\}=\sin ^{-1} \rho, \quad \text { or } \quad \theta_{m}(t)+\frac{v_{m}(N-1)(1+\rho)}{r_{l b}} \delta \leq \theta_{m}(t)+\frac{v_{m}(N-1)(1+\rho)}{r_{l b}} \delta^{*}
\end{aligned}
$$

since the sine function is monotonous for $\theta_{m}(t) \in\left[\cos ^{-1} \rho, \sin ^{-1} \rho\right]$ for $t>T_{2}$. Hence,

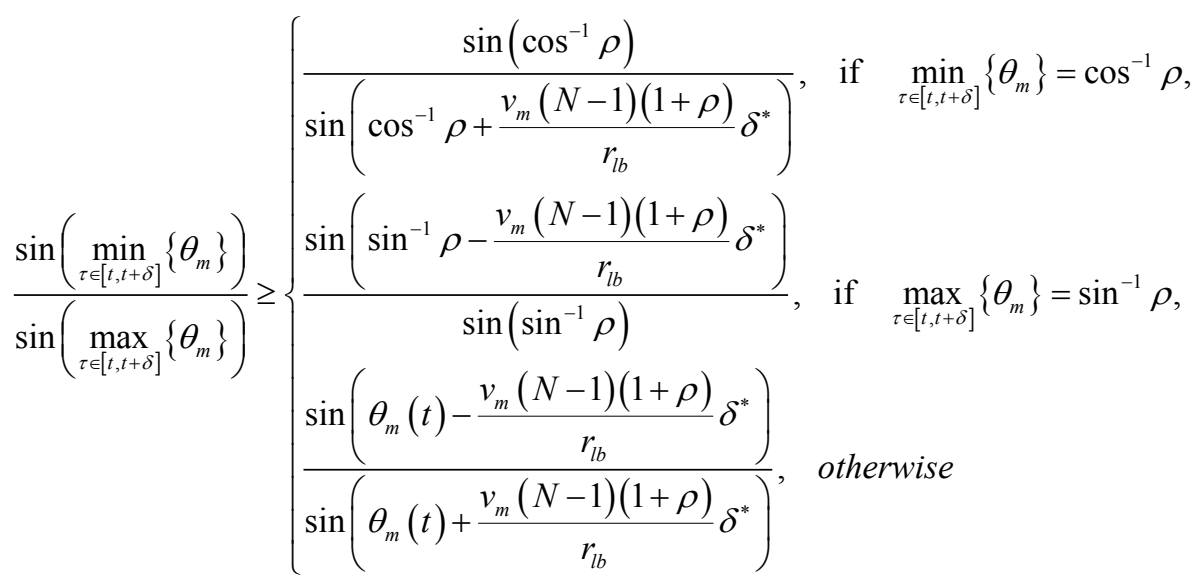

Combining (85) and (89) leads to

$$
\frac{\min _{\tau \in[t, t+\delta]}\left\{\sin \theta_{m}\right\}}{\max _{\tau \in[t, t+\delta]}\left\{\sin \theta_{m}\right\}} \geq \alpha, \quad\left\{\begin{array}{l}
\forall t \geq T_{2} \\
\forall \delta \in\left(0, \delta^{*}\right]
\end{array}\right.
$$

In the same way, the requirement on $\delta^{*}$ can also be found to make

$$
\frac{\min _{\tau \in[t, t+\delta]}\left\{\cos \theta_{m}\right\}}{\max _{\tau \in[t, t+\delta]}\left\{\cos \theta_{m}\right\}} \geq \alpha, \quad\left\{\begin{array}{l}
\forall t \geq T_{2} \\
\forall \delta \in\left(0, \delta^{*}\right]
\end{array}\right.
$$

Therefore, it has

$$
\min \left\{\frac{\min _{\tau \in[t, t+\delta]}\left(\sin \theta_{m}\right)}{\max _{\tau \in[t, t+\delta]}\left(\sin \theta_{m}\right)}, \quad \frac{\min _{\tau \in[t, t+\delta]}\left(\cos \theta_{m}\right)}{\max _{\tau \in[t, t+\delta]}\left(\cos \theta_{m}\right)}\right\} \geq \alpha, \quad\left\{\begin{array}{l}
\forall t \geq T_{2} \\
\forall \delta \in\left(0, \delta^{*}\right]
\end{array}\right.
$$

for a properly chosen $\delta^{*}$.

Besides, (69), (82), Lemma 6 and 7, and the proof of Theorem 1 under the condition that $S$ is finite ((75) (81)) indicate that, $\left\{\prod_{i=1}^{n} \prod_{k=0}^{\substack{k_{i}-1, i \neq n \\ j, i n}} \phi_{i k}, j=0,1,2,3, \ldots\right\}$ is an infinite sequence, which is decreasing and converging to a constant. Thus, it is obvious that 
$\phi_{n k} \rightarrow 1$ as $k \rightarrow \infty$. Then, according to the characteristics of $\Phi\left(t_{1}, t_{2}\right) \Phi\left(t_{2}, t_{1}\right)$ which have been already discussed by $((21) \sim(23)$ in [10]), $\phi_{n k} \rightarrow 1$ as $k \rightarrow \infty$ with (72) indicates that

$$
\left|\theta_{m}\left(\tau_{n(j+1)}\right)-\theta_{m}\left(\tau_{n j}\right)\right| \rightarrow 0, \text { as } \quad j \rightarrow+\infty
$$

which means, $\left\{\theta_{m}\left(\tau_{n j}\right), j=0,1,2, \ldots\right\}$ is a Cauchy sequence. Since the sequence is defined on a complete space $S$, there exists a constant $\theta_{m}^{*}$ that

$$
\theta_{m}\left(\tau_{n j}\right) \rightarrow \theta_{m}^{*}, \quad \text { as } \quad j \rightarrow+\infty
$$

According to (77),

$$
\theta_{m}^{*} \in\left[\cos ^{-1} \rho, \sin ^{-1} \rho\right]
$$

which means

$$
\sqrt{1-\rho^{2}} \leq \sin \theta_{m}^{*} \leq \rho, \quad \cos \theta_{m}^{*} \leq \rho<\rho / \alpha
$$

According to (92), there exists a time constant $T_{4} \geq T_{2}$ that

$$
\alpha \sin \theta_{m}^{*} \leq \sin \theta_{m}(t) \leq \frac{\sin \theta_{m}^{*}}{\alpha}, \quad t \geq T_{4}
$$

Then, (67), (94) and (96) indicate that, for a given constant $\varepsilon_{2}$ which satisfies

$$
\begin{gathered}
\varepsilon_{2}<\min \left\{\sin \theta_{m}^{*}-\sqrt{1-\rho^{2}}, \rho-\sin \theta_{m}^{*},(1-\alpha) \sqrt{1-\rho^{2}} / \alpha\right\} \\
\left|\sin \theta_{m}^{*}-\sin \theta_{m}(t)\right| \leq \varepsilon_{2}, \quad t \geq T_{4}
\end{gathered}
$$

On the other hand, (67) and (94) also imply that, for any given positive constant $\varepsilon_{3}$, there exists a time constant $T_{5}$ such that, for any constant $\delta$,

$$
\left|\theta_{m}\left(T_{5}+k^{\prime \prime} \delta\right)-\theta_{m}\left(T_{5}+\left(k^{\prime \prime}-1\right) \delta\right)\right| \leq \varepsilon_{3}, \quad k^{\prime \prime}=1,2, \ldots
$$

Thus, there exists a time constant $T_{6}$ such that

$$
\begin{aligned}
& \left|\theta_{m}\left(T_{6}+k^{\prime \prime} \delta^{*}\right)-\theta_{m}\left(T_{6}+\left(k^{\prime \prime}-1\right) \delta^{*}\right)\right| \\
& \leq\left[\alpha-\sqrt{\rho^{2}+\gamma^{2}}\right](N-1) v_{m} \delta^{*} / r_{\text {max }},
\end{aligned}
$$

where $r_{\max }$ is given in (49) and $\gamma$ is a positive constant satisfying

$$
\gamma \in\left(0, \alpha \sqrt{\rho^{2}-\left(\varepsilon_{2}+\sin \theta_{m}^{*}\right)^{2}}\right)
$$

According to (98) and (102), a proper $\alpha \in(\rho, 1)$ can be chosen to satisfy

$$
\alpha-\sqrt{\rho^{2}+\gamma^{2}}>0
$$


Now, let $T^{*}=\max \left\{T_{4}, T_{6}\right\}$. Then, (99) and (101) can be converted into

$$
\begin{gathered}
\left|\sin \theta_{m}^{*}-\sin \theta_{m}(t)\right| \leq \varepsilon_{2}, \quad t \geq T^{*} \\
\left|\theta_{m}\left(T^{*}+k^{\prime \prime} \delta^{*}\right)-\theta_{m}\left(T^{*}+\left(k^{\prime \prime}-1\right) \delta^{*}\right)\right| \\
\leq\left[\alpha-\sqrt{\rho^{2}+\gamma^{2}}\right](N-1) v_{m} \delta^{*} / r_{\max },
\end{gathered}
$$

So far, some characteristics of $\theta_{m}(t)$ under (82) have been given. Next, the upper bound of $\int\left[\cos \theta_{t}(t) / r(t)\right] \mathrm{d} t$ on an infinite series of equal-length intervals will be deduced.

Let $\Lambda_{k^{\prime \prime}}=\left[T^{*}+\left(k^{\prime \prime}-1\right) \delta^{*}, T^{*}+k^{\prime \prime} \delta^{*}\right]$. Then, by integrating (39), along with (105), we have

$$
\left|\int_{\Lambda_{k^{\prime}}} \frac{\rho \sin \theta_{t}(t) \cos \Theta(t)}{r(t)} \mathrm{d} t-\int_{\Lambda_{k^{\prime}}} \frac{\sin \theta_{m}(t)}{r(t)} \mathrm{d} t\right| \leq \frac{\alpha-\sqrt{\rho^{2}+\gamma^{2}}}{r_{\max }} \delta^{*}
$$

from (106) we have

$$
\begin{aligned}
\int_{\Lambda_{k^{\prime}}} \frac{\rho \sin \theta_{t}(t)}{r(t)} \mathrm{d} t & \geq \int_{\Lambda_{k^{\prime}}} \frac{\rho \sin \theta_{t}(t)|\cos \Theta(t)|}{r(t)} \mathrm{d} t \\
& \geq \int_{\Lambda_{k^{\prime \prime}}} \frac{\sin \theta_{m}(t)}{r(t)} \mathrm{d} t-\frac{\alpha-\sqrt{\rho^{2}+\gamma^{2}}}{r_{\max }} \delta^{*}
\end{aligned}
$$

Note that $r(t)$ and $\theta_{m}(t)$ are continuous functions of time. There must exist a constant time $t_{k^{\prime \prime}}^{*} \in \Lambda_{k^{\prime \prime}}$ that

$$
\int_{\Lambda_{k^{\prime}}}[1 / r(t)] \mathrm{d} t=\delta^{*} / r\left(t_{k^{*}}^{*}\right)
$$

By this, along with (92), we have

$$
\begin{aligned}
& \int_{\Lambda_{k^{\prime}}}\left[\sin \theta_{m}(t) / r(t)\right] \mathrm{d} t \geq \alpha \delta^{*} \sin \theta_{m}\left(t_{k^{\prime \prime}}^{*}\right) / r\left(t_{k^{\prime \prime}}^{*}\right) \\
& \int_{\Lambda_{k^{\prime \prime}}}\left[\cos \theta_{m}(t) / r(t)\right] \mathrm{d} t \geq \alpha \delta^{*} \cos \theta_{m}\left(t_{k^{\prime \prime}}^{*}\right) / r\left(t_{k^{\prime \prime}}^{*}\right)
\end{aligned}
$$

Then, it follows from (49), (107), and (109) that

$$
\int_{\Lambda_{k^{\prime}}} \frac{\rho \sin \theta_{t}(t)}{r(t)} \mathrm{d} t \geq \alpha \delta^{*} \frac{\sin \theta_{m}\left(t_{k^{\prime \prime}}^{*}\right)}{r\left(t_{k^{\prime \prime}}^{*}\right)}-\frac{\alpha-\sqrt{\rho^{2}+\gamma^{2}}}{r_{\max }} \delta^{*} \geq\left[\alpha \sin \theta_{m}\left(t_{k^{\prime \prime}}^{*}\right)-\alpha+\sqrt{\rho^{2}+\gamma^{2}}\right] \frac{\delta^{*}}{r\left(t_{k^{\prime \prime}}^{*}\right)}, \quad k^{\prime \prime}=1,2, \ldots
$$

Using the Schwartz inequality, it can be shown that

$$
\left|\int_{\Lambda_{k^{\prime}}} \frac{\cos \theta_{t}(t)}{r(t)} \mathrm{d} t\right| \leq \sqrt{\int_{\Lambda_{k^{\prime}}} \frac{1-\sin \theta_{t}(t)}{r(t)} \mathrm{d} t \cdot \int_{\Lambda_{k^{\prime}}} \frac{1+\sin \theta_{t}(t)}{r(t)} \mathrm{d} t}=\sqrt{\left[\int_{\Lambda_{k^{\prime}}} \frac{1}{r(t)} \mathrm{d} t\right]^{2}-\left[\int_{\Lambda_{k^{\prime}}} \frac{\sin \theta_{t}(t)}{r(t)} \mathrm{d} t\right]^{2}}
$$

which along with (108) (110) indicates that 


$$
\int_{\Lambda_{k^{\prime}}} \frac{\rho \cos \theta_{t}(t)}{r(t)} \mathrm{d} t \leq\left|\int_{A_{k^{\prime}}} \frac{\rho \cos \theta_{t}(t)}{r(t)} \mathrm{d} t\right| \leq \sqrt{\left[\int_{\Lambda_{k^{\prime}}} \frac{\rho}{r(t)} \mathrm{d} t\right]^{2}-\left[\int_{\Lambda_{k^{\prime}}} \frac{\rho \sin \theta_{t}(t)}{r(t)} \mathrm{d} t\right]^{2}} \leq \frac{\sqrt{\rho^{2}-\left[\alpha \sin \theta_{m}\left(t_{k^{\prime \prime}}^{*}\right)-\alpha+\sqrt{\rho^{2}+\gamma^{2}}\right]^{2}}}{r\left(t_{k^{\prime \prime}}^{*}\right)}
$$

Now, we come to the final step of the proof, which shows that in the time sequence $\Lambda_{k^{n}}, r$ converges to zero. According to (28), (96), and (112), we have

$$
\begin{aligned}
\frac{1}{v_{m}} \int_{A^{*}} \frac{\dot{r}(t)}{r(t)} \mathrm{d} t & =\left[\int_{A_{k^{\prime}}} \frac{\rho \cos \theta_{t}(t)}{r(t)} \mathrm{d} t-\int_{\Lambda_{k^{\prime}}} \frac{\cos \theta_{m}(t)}{r(t)} \mathrm{d} t\right] \leq\left\{\sqrt{\rho^{2}-\left[\alpha \sin \theta_{m}\left(t_{k^{*}}^{*}\right)-\alpha+\sqrt{\rho^{2}+\gamma^{2}}\right]^{2}}-\alpha \cos \theta_{m}\left(t_{k^{*}}^{*}\right)\right\} \frac{\delta^{*}}{r\left(t_{k^{*}}^{*}\right)} \\
& =-\frac{2 \alpha\left(\alpha-\sqrt{\rho^{2}+\gamma^{2}}\right)\left[1-\sin \theta_{m}\left(t_{k^{\prime \prime}}^{*}\right)\right]+\gamma^{2}}{\sqrt{\rho^{2}-\left[\alpha \sin \theta_{m}\left(t_{k^{\prime \prime}}^{*}\right)-\alpha+\sqrt{\rho^{2}+\gamma^{2}}\right]^{2}}+\alpha \cos \theta_{m}\left(t_{k^{\prime \prime}}^{*}\right)} \frac{\delta^{*}}{r\left(t_{k^{\prime}}^{*}\right)}<-\frac{\gamma^{2} \delta^{*}}{r_{\max }\left[\sqrt{\alpha^{2}-\gamma^{2}}+\rho\right]}
\end{aligned}
$$

It can be easily seen from the above inequality that the sequence $\left\{r\left(T_{6}+k^{\prime \prime} \delta^{*}\right), k^{\prime \prime}=1,2, \ldots\right\}$ converges exponentially to zero, which contradicts (82). Therefore, Theorem 1 holds under this situation.

The original formations of (79) (94), (99) (113) can be found in (Proof (of the sufficient part of Theorem 1) in [10]). However, comparing the above proof process with the counterpart in [10] it can be seen that, for intercepting lower-speed arbitrarily maneuvering targets satisfying (27), 3D PPN with $N>1$ will enter the range of $\theta_{m} \leq \sin ^{-1} \rho$ ultimately, and $\dot{r} \geq 0$ only happens in the range of $\theta_{m} \in\left[\cos ^{-1} \rho, \sin ^{-1} \rho\right]$. Besides, all variables concerned in the above proof of Theorem 1 are in 3D space, which is a progress compared with the previous result.

\section{Simulation Results}

For the length of this paper, we just adopt the initial engagement geometry of $\theta_{m}(0)>\sin ^{-1} \rho$ in this section. And $v_{m}$ and $v_{t}$ are properly chosen to meet $\rho>1 / \sqrt{2}$.

The simulation model is constructed in accordance with the assumption of ideal relative dynamics between the missile and target, as stated in Section II. The sampling period $T=10 \mathrm{~ms}$ is selected before the relative range $r<500 \mathrm{~m}$. After that $T$ is chosen to be $0.1 \mathrm{~ms}$. When $r<r_{\min }=0.1 \mathrm{~m}$, we think the intercept happens and the simulation is stopped. Actually, if a smaller $T$ is chosen after $r<500 \mathrm{~m}, r_{\min }$ could be much smaller than $0.1 \mathrm{~m}$. The simulation data are shown in Tab. 1 .

TABLE 1

SiMULATION DATA

\begin{tabular}{clc}
\hline \hline Symbol & \multicolumn{1}{c}{ Quantity } & Value \\
\hline$r_{0}$ & Initial relative distance & $5000 \mathrm{~m}$ \\
$v_{m}$ & Missile speed & $500 \mathrm{~m} / \mathrm{s}$ \\
$v_{t}$ & Target speed & $400 \mathrm{~m} / \mathrm{s}$
\end{tabular}




$\begin{array}{llr}\varphi_{L 0} & \text { Initial LOS elevation angle } & 20^{\circ} \\ \psi_{L 0} & \text { Initial LOS azimuth angle } & 120^{\circ} \\ \boldsymbol{r}_{m 0} & \text { Initial position of missile } & {[0,0,10000] \mathrm{m}} \\ \varphi_{t 0} & \text { Euler angles from LOS coordinate } & -20^{\circ} \\ & \text { system to target body coordinate } & 00^{\circ} \\ \psi_{t 0} & \text { system } & 30^{\circ} \\ \varphi_{m 0} & \text { Euler angles from LOS coordinate } & \\ & & \\ \psi_{m 0} & \text { system to missile body coordinate } & 60^{\circ} \\ & \text { system } & \\ \theta_{m 0} & \text { Initial lead angle of missile } & \end{array}$

3D PPN of (8) is used to guide the missile. According to the prior result of (32) (Theorem 2 in [9]), under these intercept situations, the navigation gain $N$ should be

$$
N>1+\sqrt{2 \rho^{2}-1} / \rho \approx 1.6614
$$

In this section, we choose $N=1.2$ to demonstrate Theorem 1.

According to Tab. 1 , the speed ratio $\rho=0.8>1 / \sqrt{2}$, then $\sin ^{-1} \rho=53.1301^{\circ}$ and $\cdot \cos ^{-1} \rho=36.8699^{\circ}$. Two kinds of target maneuver are considered. The first one is

$$
\boldsymbol{A}_{T}=100\left(\boldsymbol{i}_{I} \times \boldsymbol{i}_{T}\right)
$$

The second one is a sinusoidal maneuver, i.e.,

$$
\boldsymbol{A}_{T}=100 \sin \left(\pi T+\frac{\pi}{6}\right)\left(\boldsymbol{i}_{I} \times \boldsymbol{i}_{T}\right)
$$

The 3D trajectories of the missile and target of both cases are shown in Fig. 5. It can be seen that, the missile guided by 3D PPN with $N=1.2$ can intercept both of the targets.

Simulation results are shown in the Figs. 5 7. 


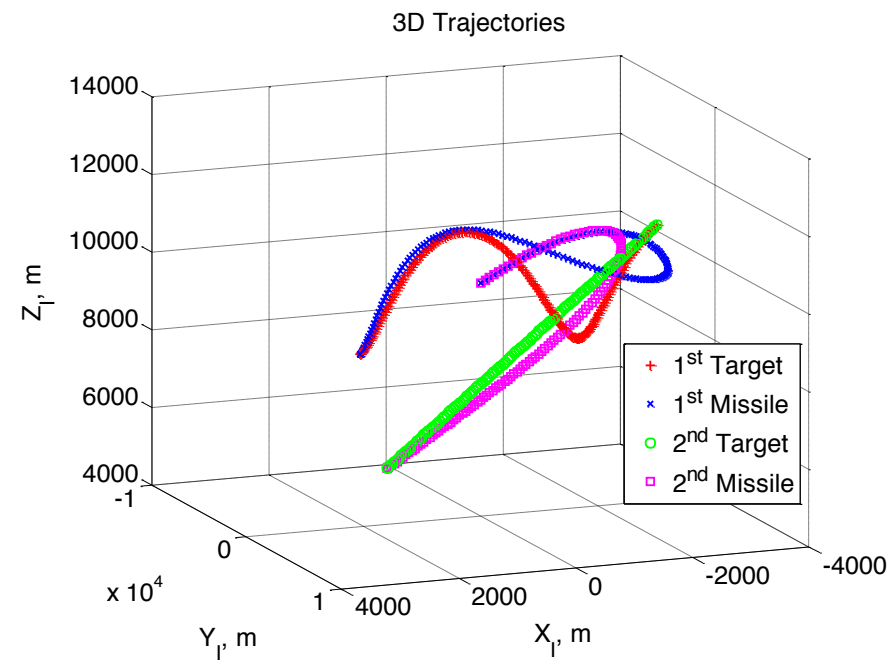

Fig. 5. Three-dimensional engagement geometries.

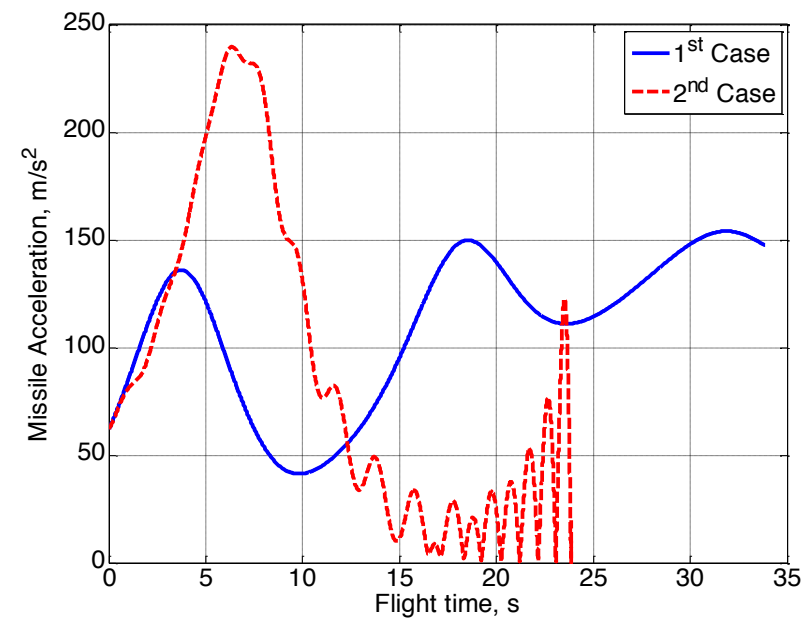

Fig. 6. Missile accelerations.

The missile accelerations of 3D PPN guidance law against both targets are shown in Fig. 6. It can be seen that, for both cases, there is a peak of $\left|\boldsymbol{A}_{M}\right|$ in the end phase of the guidance process. For the first kind of target, the interception time is longer and the peak value of $\left|\boldsymbol{A}_{M}\right|$ is smaller. For the second kind of target, the curve of $\left|\boldsymbol{A}_{M}\right|$ is wavy before the end phase, which is caused by the sinusoidal maneuver of the target. In both cases, it can be seen that $\left|\boldsymbol{A}_{M}\right|$ sometimes becomes very high. This is impractical for any missile. In reality the overload of the missile will be constrained by a saturation value. And if the practical guidance and control system of the missile cannot provide enough acceleration to match the guidance command, the capturability of the guidance law cannot be guaranteed and the miss distance will be introduced.

The closing speeds of both cases are shown in the above subfigure of Fig. 7. It can be seen that, for the first kind of target, there is a time interval where $\dot{r}>0$ happens during the guidance process. For the second kind of target, the closing speed is always 
minus. The missile lead angles are shown in the below subfigure of Fig. 7, from which we can see that, Lemma 1 is valid and well satisfied for both cases.
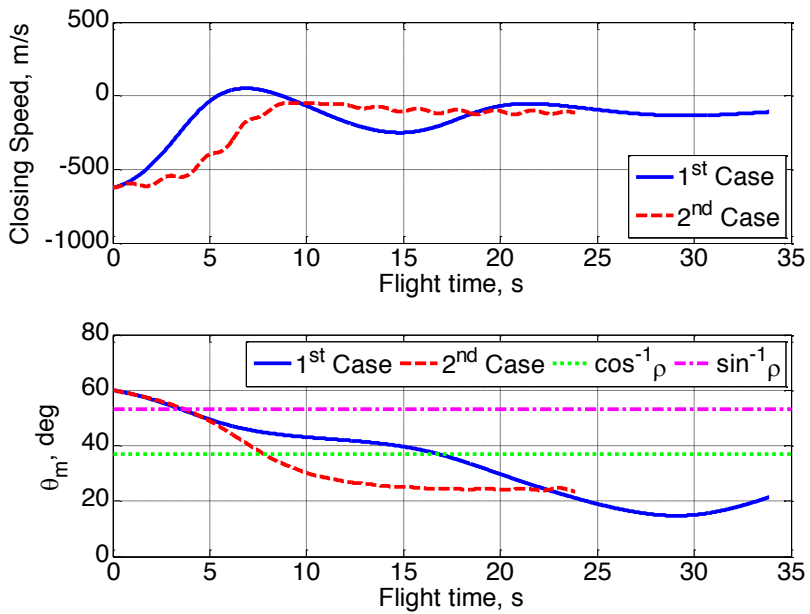

Fig. 7. Closing speeds and lead angles.

\section{CONCLUSION}

This work has extended the NOR method of 2D PPN to 3D space based on the relative kinematic equation established in the rotating LOS coordinate system, and has filled the gap between the capturability analysis result of 2D PPN and that of 3D PPN against lower-speed arbitrarily maneuvering targets. It is proven that, if the navigation gain is larger than one, 3D PPN can capture the lower-speed arbitrarily maneuvering target for the homing phase of the missile. The future work may need to focus on the capturability analysis of 3D PPN against higher-speed arbitrarily maneuvering targets, which has not been conducted completely and perfectly thus far.

\section{REFERENCES}

[1] Shneydor, N. A., "Missile guidance and pursuit - kinematics, dynamics and control," Chichester: Horwood Publishing, 1998.

[2] Guelman, M., “A qualitative study of proportional navigation,” IEEE Transactions on Aerospace and Electronic Systems. 1971, 7(4): 637643.

[3] Guelman, M., "Proportional navigation with a maneuvering target," IEEE Transactions on Aerospace and Electronic Systems. 1972, 8(3): 364-371.

[4] Guelman. M., "Missile acceleration in proportional navigation," IEEE Transactions on Aerospace and Electronic Systems. 1973, 9(5): 462463.

[5] Becker, K., "Closed-form solution of pure proportional navigation, ” IEEE Transactions on Aerospace and Electronic Systems. 1990, 26(3): 526-533. 
[6] Ghawghawe, S. N., and Ghose, D., "Pure proportional navigation against time-varying target maneuvers," IEEE Transactions on Aerospace and Electronic Systems. 1996, 32(4): 1336-1347.

[7] Ha, I. J., Hur, J. S., Ko, M. S., and Song, T. L., "Performance analysis of PNG laws for randomly maneuvering targets," IEEE Transactions on Aerospace and Electronic Systems. 1990, 26(5): 713-721.

[8] Song, S. H., and Ha, I. J., “A lyapunov-like approach to performance analysis of 3-dimension pure PNG laws,” IEEE Transactions on Aerospace and Electronic Systems. 1994, 30(1), 238-248.

[9] Oh, J. H., and Ha, I. J., “Capturability of the three-dimensiona pure PNG Law," IEEE Transactions on Aerospace and Electronic Systems. 1999, 35(2): 491-503.

[10] Oh, J. H., “Solving a nonlinear output regulation problem: zero miss distance of pure PNG,” IEEE Transactions on Automatic Control. 2002, 47(1): 169-173.

[11] Li, K. B., Chen, L., and Bai, X. Z., "Differential geometric modeling of guidance problem for interceptors,” Sci China Tech Sci, 2011, 54(9): 2283-2295.

[12] Li, K. B., Chen, L., and Tang, G. J., "Improved differential geometric guidance commands for endoatmospheric interception of high-speed targets,” Sci China Tech Sci, 2013, 56(2): 518-528.

[13] Li, K. B., Chen, L., and Tang, G. J., “Algebraic solution of differential geometric guidance command and time delay control,” Sci China Tech Sci, 2015, 58(3): 565-573.

[14] Shin, H. S., Tsourdos, A., Li, K. B., “A new three-dimensional sliding mode guidance law variation with finite time convergence,” IEEE Transactions on Aerospace and Electronic Systems, 2017, 53(5): 2221-2232.

[15] Li, K. B., Liang, Y. G., Su, W. S., and Chen, L., "Performance of 3D TPN against true-arbitrarily maneuvering target for exoatmospheric interception,” Sci China Tech Sci, 2018, 61(8): 1161-1174.

[16] Tyan, F., "Unified approach to missile guidance laws: a 3D extension,” IEEE Transactions on Aerospace and Electronic Systems, 2005, 41(4): 1178-1199.

[17] Tyan, F., "Capture region of a GIPN guidance law for missile and target with bounded maneuverability," IEEE Transactions on Aerospace and Electronic Systems, 2011, 47(1): 201-213.

[18] Ghosh, S., Ghose, D., and Raha, S., "Capturability analysis of a 3-D retro-PN guidance law for higher speed nonmaneuvering targets," IEEE Transactions on Control Systems Technology. 2014, 22(5): 1864-1874.

[19] Tyan, F., "Capture region of 3D PPN guidance law for intercepting high Speed target," Joint 48th Conference on Decision and Control and 28th Chinese Control Conference, Shanghai, China, December 16-18, 2009.

[20] Tyan, F., "Capture region of PPN guidance law with observe lag,” Proceedings of 2011 8th Asian Control Conference (ASCC), Kaohsiung, Taiwan, May 15-18, 2011, pp. 465-469.

[21] Tyan, F., “Analysis of 3D PPN Guidance Laws for Nonmaneuvering Target,” IEEE Transactions on Aerospace and Electronic Systems, 2015, 51(4): 29322-9432. 
[22] Ghosh, S., Ghose, D., and Raha, S., “Composite Guidance for Impact Angle Control Against Higher Speed Targets,” Journal of Guidance, Control, and Dynamics, 2016, 39(1): 98-117. 
2019-08-30

\title{
Capturability of 3D PPN against
}

lower-speed maneuvring target for

homing phase

\author{
Li, Ke-Bo
}

IEEE

Li K-B, Shin H-S, Tsourdos A, Tahk M-J. (2020) Capturability of 3D PPN against lower-speed maneuvring target for homing phase. IEEE Transactions on Aerospace and Electronic Systems, Volume 56, Issue 1, February 2020, pp. 711-722

https://doi.org/10.1109/TAES.2019.2938601

Downloaded from Cranfield Library Services E-Repository 